\title{
The Price Equation and Extended Inheritance
}

\author{
Heikki Helanterä $§$ and Tobias Uller $\ddagger$
}

\begin{abstract}
The presence of various mechanisms of non-genetic inheritance is one of the main problems for current evolutionary theory according to several critics. Sufficient empirical and conceptual reasons exist to take this claim seriously, but there is little consensus on the implications of multiple inheritance systems for evolutionary processes. Here we use the Price Equation as a starting point for a discussion of the differences between four recently proposed categories of inheritance systems; genetic, epigenetic, behavioral and symbolic. Specifically, we address how the components of the Price Equation encompass different non-genetic systems of inheritance in an attempt to clarify how the different systems are conceptually related. We conclude that the four classes of inheritance systems do not form distinct clusters with respect to their effect on the rate and direction of phenotypic change from one generation to the next in the absence or presence of selection. Instead, our analyses suggest that different inheritance systems can share features that are conceptually very similar, but that their implications for adaptive evolution nevertheless differ substantially as a result of differences in their ability to couple selection and inheritance.
\end{abstract}

\section{KEYWORDS \\ Behavioral inheritance $\bullet$ Cultural evolution $\bullet$ Epigenetic inheritance \\ - Horizontal transmission • Modern Synthesis • Parental effects}

We should not expect a single, universal model for ... all the dimensions of heredity and evolution — E. Jablonka \& M.J. Lamb $(2005,378)$

This expanded Price Equation provides an exact description of total evolutionary change under all conditions, and for all systems of inheritance and selection

- S. Frank $(1997,1712)$

Despite the statement by Jablonka and Lamb quoted above, evolutionary theorists tend to agree with Frank that there is a unifying mathematical formulation of evolutionary change, known as the Price Equation or Price Theorem (Frank 1995, 1997; Price 1970, 1972; Rice 2004). This equation has been instrumental for the development of evolutionary theory, in particular with respect to kin and multi-level selection (Frank 1998; Gardner 2008; Okasha 2006). The power of the Price Equation is that it does not make any assumptions regarding the kind of entities that evolve or the mechanisms of inheritance. Consequently, the Price Equation could provide a framework for comparing evolution under different types of inheritance mechanisms, and thereby quantify the implications of non-genetic inheritance for

$\S$ Department of Biological and Environmental Sciences, University of Helsinki, POB 65, FI-00014, Finland ‡ Department of Zoology, The Tinbergen Building, South Parks Road, Oxford, OX1 3PS, UK

E-mail: heikki.helantera@helsinki.fi

Received 7 October 2009; Accepted 18 November 2009 
evolutionary theory. In this paper, we explore to what extent the Price Equation can help us conceptualize differences between inheritance systems and illustrate their effects on the rate and direction of phenotypic change.

\section{Systems of Inheritance}

In a biological context, inheritance describes the processes that cause reliable recurrence of phenotypic traits within lineages (Mameli 2005). Despite the fact that the theory of evolution by natural selection was conceived in the absence of empirical knowledge about the mechanism of inheritance, inheritance is a central concept in evolutionary theory (Mameli 2005; Mayr 1982). With the development of the Modern Synthesis, inheritance has become almost synonymous with the transmission of genetic material from parents to offspring (Mayr 1982; Sarkar 2006). In other words, both stability of phenotypic traits across generations within lineages and consistent differences between lineages have their origin in the genome (Mameli 2004, 2005). Nevertheless, it is quite obvious that there is more to inheritance than transgenerational transmission of genetic material (Badyaev and Uller 2009; Boyd and Richerson 1985; CavalliSforza and Feldman 1981; Jablonka and Lamb 2005; Odling-Smee et al. 2003; Oyama et al. 2001). For example, we are all familiar with several types of non-genetic inheritance, such as cultural inheritance of social status and wealth, and similar types of trans-generational transmission of resources occur also in nonhuman animals (Avital and Jablonka 2000). In addition, cultural phenotypes need not be inherited from the biological parents, but through horizontal transmission from other members of the population (Boyd and Richerson 1985; Cavalli-Sforza and Feldman 1981). Importantly, several authors have claimed that the presence of non-genetic inheritance not only questions the prevailing description of trans-generational stability of phenotype, but also that it has implications for our understanding of the process of evolution itself (Badyaev and Uller 2009; Boyd and Richerson 1985; Jablonka and Lamb 1995, 2005; Odling-Smee et al. 2003; Oyama et al. 2001).

In a forceful argument for the developmental and evolutionary importance of extended inheritance, Jablonka and Lamb (2005) recently proposed a separation of inheritance systems ('dimensions') into four different classes: genetic, epigenetic, behavioral, and symbolic inheritance. This classification seems to have been largely accepted and quite influential, but it is unclear whether it captures the importance of extended inheritance for evolutionary processes. Our aim here is therefore to attempt to conceptually unify different inheritance systems under the framework of the Price Equation. We briefly summarize the four inheritance systems as classified by Jablonka and Lamb before addressing whether the Price Equation can help us understand how they are related conceptually to each other and to other frameworks where non-genetic inheritance has been considered.

\subsection{Genetic inheritance}

Genetic inheritance, which lies at the heart of the Modern Synthesis (Mayr and Provine 1980), is based on transmission of genetic material (i.e., DNA) from parents to offspring and occurs in all organisms (Maynard Smith and Szathmáry 1995). The relationship between genotype and phenotype under genetic inheritance may be complex due to, for example, epistatic effects, phenotypic plasticity or indirect genetic effects (Pigliucci 2001; Wolf et al. 1998; Wolf et al. 2000). Nevertheless, in the absence of other means of transmission of form, recurrence of phenotypes across generations within lineages and consistent differences between lineages can indeed be traced back to the genome and adaptive evolution can therefore be described as changes in gene frequencies. Origin of novel phenotypes relies upon mutation, recombination, and the fusion of genetic material from different individuals through, for example, sexual reproduction (Futuyma 2005). While most of the Modern Synthesis is built on vertical transmission, genetic material can also be transmitted horizontally (Ochman et al. 2000). Furthermore, instead of occurring completely at random, mutation in micro-organisms may be induced by external conditions (Bjedov et al. 2003; Ochman et al. 2000; Tenaillon et al. 2004) and perhaps target certain parts of the genome and thereby cause some form of directionality (Caporale 2000). Both of these possibilities call for extending the Modern Synthesis view of genetic inheritance (Jablonka and Lamb 2005). 


\subsection{Epigenetic inheritance}

Epigenetic inheritance is a very broad term that essentially includes many different phenomena both between cell generations during individual development, and between phenotypes across generations of individuals (Jablonka and Lamb 1995). Here we are concerned with cellular epigenetic inheritance, defined as transmission from mother cell to daughter cell of variations that are not the result of DNA differences (Jablonka and Raz 2009). Below, we will further restrict our discussion to cellular epigenetic inheritance across generations via meiotic divisions in multicellular organisms. For example, eukaryotic genomes are modified by structures (chromatin marks, e.g., methylation patterns) that affect gene expression and variation in chromatin marks can lead to large phenotypic variation (Allis et al. 2007; Rando and Verstrepen 2007). One of the most interesting features of epigenetic inheritance is that changes in chromatin marks can be induced by environmental conditions (e.g., via exposure to chemicals) and be transferred to the offspring (Crews et al. 2007; Molinier et al. 2006; Rando and Verstrepen 2007; Richards 2006). Thus, they provide an opportunity for environmentally induced phenotypic changes to reoccur in future generations in the absence of genetic change. A classic example of epigenetic inheritance is coat color in mice, where variation in methylation patterns of a mutated part of the DNA gives rise to variation in coat colors that are stably inherited by the offspring in the absence of genetic variation (Morgan et al. 1999). Similar phenomena have also been described in plants (Cubas et al. 1999; Martienssen and Colot 2001; Rapp and Wendel 2005). The stability of epigenetic inheritance in natural populations is still unknown, as is the importance of environmental conditions for maintenance of chromatin marks across generations (Bossdorf et al. 2008; Richards 2006).

\subsection{Behavioral inheritance}

The behavioral inheritance system is defined as the transmission of a phenotype across generations that involve interactions with other individuals (Jablonka and Lamb 2005). While this definition potentially covers several diverse phenomena involved in cultural evolution and social learning (see below, Section 4.5: Avital and Jablonka 2000; Boyd and Richerson 1985; Boyd and Richerson 2005; Cavalli-Sforza and Feldman 1981; Franz and Nunn 2009; Laland 2004), Jablonka and Lamb (2005) recognize three different mechanisms. The first refers to the case where development of offspring traits are affected by maternal transfer of non-nucleic acid substances, such as when maternal diet during lactation influences the development of food preferences in the offspring (Bilkó et al. 1994). The second refers to learning from other individuals via imitation. The third, non-imitative learning, is similar to the second, but in this case there is no direct imitation involved and trait recurrence is enabled via trial and error; the trials themselves are stimulated by observation of other individuals. For example, bumblebees learn how to rob nectar by biting through the base of the flower when they forage on flowers which have been robbed by other bumblebees (Leadbeater and Chittka 2008). An important distinction between the first versus the second and third is that the latter two can occur horizontally, whereas the former relies upon a more or less intimate parent-offspring relationship. While Jablonka and Lamb (2005) discuss horizontal transmission mainly in terms of learning, there are also other cultural phenomena that lead to horizontal transmission of traits, including coercive and conformist strategies (Boyd and Richerson 1985, 2005; Cavalli-Sforza and Feldman 1981; Ehrlich and Levin 2005; Henrich and Boyd 1998).

\subsection{Symbolic inheritance}

The final class of inheritance mechanisms identified by Jablonka and Lamb is symbolic inheritance. This includes language and cultural practices that transfer from an individual to another through the use of symbols and are therefore not fully captured under the behavioral inheritance system tag. The most important features of this inheritance system that sets it apart from the other is that symbolic inheritance allows for: (a) latency, i.e., acquisition of traits from a larger array than just those present in the individuals of the parental generation through the existence of symbolic instructions; (b) the transmission of traits without 
the individuals themselves interacting; and, (c) inherited instructions to be "planned and constructed" (Jablonka and Lamb 2005; Maynard Smith and Szathmáry 1995).

\section{Implications of Different Inheritance Systems for Evolutionary Theory}

Some claim that the presence of multiple modes of inheritance poses a significant threat to current evolutionary thought (Boyd and Richerson 1985; Jablonka and Lamb 2005; Jablonka and Raz 2009; Odling-Smee et al. 2003; Varmuza 2003). For our purposes, it is useful to split these challenges into two. First is the challenge to the traditional view from molecular genetics that only nucleic acids transmit information between generations (Crick 1970). It is clear from the ample literature on cultural and epigenetic inheritance that this challenge is justified (see above). Second, there is the challenge that nongenetic inheritance would force a revision of evolutionary theory (Jablonka and Lamb 2005; Pigliucci 2007). The latter challenge does not necessarily follow from the former. Several authors (e.g., Gorelick and Laubichler 2008; Haig 2007), have pointed out that the foundations of the Modern Synthesis in the works of Fisher, Wright, and others, were constructed without detailed information on the substance of hereditary material (see also Mayr 1982; Provine 2001). Thus, as long as offspring inherit determinants of phenotype and fitness from their parents, the theoretical basis of the Modern Synthesis is not dependent on a specific kind of hereditary material, such as DNA.

The extent of the latter challenge is yet to be fully assessed (Pigliucci 2007). We admit there are several ways, described in a large body of literature, in which different means of inheritance could affect evolutionary processes (for recent treatments see, e.g., Feldman and Laland 1996; Jablonka and Lamb 1995, 2005; Odling-Smee et al. 2003; Oyama et al. 2001; Weber and Depew 2003). Some of these are only indirectly linked to the direct effect of trait transmission across consecutive generations. For example, the presence of behavioral and symbolic inheritance systems could significantly change the conditions, and thereby selective regimes, for future generations (Boyd and Richerson 1985; Cavalli-Sforza and Feldman 1981; Odling-Smee et al. 2003; Weber and Depew 2003). However, since inheritance specifically refers to means of recurrence of phenotypes across generations, a more direct way in which inheritance systems could differ would be in their effect on the rate and direction of short term evolutionary change in the presence or absence of selection. Here we are concerned with describing short term changes in a population with respect to an optimal phenotype, the domain where Modern Synthesis is at its strongest (Rice 2004), and less with evolution on longer time scales. We try to assess evolutionary implications of different inheritance mechanisms by integrating them into mainstream evolutionary theory. More specifically, we explore to what extent a general mathematical formulation of evolutionary change, known as the Price Equation (Frank 1995; Gardner 2008; Price 1970, 1972), can assist in highlighting differences and similarities between inheritance systems described above and show how they are conceptually related.

\section{The Price Equation and Evolutionary Ghange}

The Price Equation is a mathematical statement that describes the change in a character, $\mathrm{z}$, from one generation to another (Price 1970). It does not rely upon any biological assumptions and is therefore completely general. Several recent works give excellent introductions to the derivation of the equation and its interpretation (Frank 1998; Gardner 2008; Godfrey-Smith 2009; Kerr and Godfrey-Smith 2009; Okasha 2006; Rice 2004). In its most common form, the equation is given in the form

$$
\text { (3.1) } w \Delta z=\operatorname{Cov}\left(w_{i}, z_{i}\right)+E\left(w_{i} \Delta z_{i}\right)
$$

Where, $\mathrm{w}$ is the population average of absolute fitness, $\Delta \mathrm{z}$ is the change in the population average of a trait, and $\mathrm{w}_{\mathrm{i}}$ and $\mathrm{z}_{\mathrm{i}}$ are fitness and trait value of individual $\mathrm{i}$, respectively. The first part of the right-hand side, the covariance between fitness and trait value, can be interpreted as the change in phenotypic value as a result of selection. For example, when the covariance is positive, individuals with larger $\mathrm{z}$ have higher survival and/or produce more offspring than individuals with smaller z. The second part of the right hand size is the 
expected value of the product of fitness and the average difference between parent and offspring in the absence of selection, i.e., the transmission bias or fidelity (Frank 1995; Okasha 2006). This transmission term describes (statistically) the extent to which offspring of a parent with trait value $z_{i}$ exhibit the same trait value. The closer $\Delta \mathrm{z}_{\mathrm{i}}$ is to zero the more faithfully the character value is transmitted between parents and offspring. Any systematic bias or noise in the transmission of traits between generations will therefore enter this term.

Given that the individuals of equation 3.1 are arbitrary units, they can also represent social groups. Thus, equation 3.1 can be expanded into

$$
(3.2) \mathrm{w} \Delta \mathrm{z}=\operatorname{Cov}\left(\mathrm{w}_{\mathrm{i}}, \mathrm{z}_{\mathrm{i}}\right)+\mathrm{E}\left\{\operatorname{Cov}\left(\mathrm{w}_{\mathrm{ij}}, \mathrm{z}_{\mathrm{ij}}\right)+\mathrm{E}\left(\mathrm{w}_{\mathrm{ij}} \Delta \mathrm{z}_{\mathrm{ij}}\right)\right\}
$$

where i now denotes groups of individuals $\mathrm{j}$. This expanded form consists of the first covariation term describing change due to selection among groups (or differences in mean fitness among groups), the expectation of total change, partitioned into a covariance term describing selection within groups (differences in fitness within groups), and the transmission effects within groups. Most often these groupings have been used in describing selection in a hierarchical setting (Frank 1998; Okasha 2006), but we use the grouping more generally to describe any within population variation in effects of environment on either selection or transmission of traits (see below).

Equation 3.1 can also be modified by dividing both sides with w, which generates

$$
\text { (3.3) } \Delta \mathrm{z}=\operatorname{Cov}\left(\omega_{\mathrm{i}}, \mathrm{z}_{\mathrm{i}}\right)+\mathrm{E}_{\omega \iota}\left(\Delta \mathrm{z}_{\mathrm{i}}\right)
$$

Where $\operatorname{Cov}\left(\omega_{\mathrm{i}}, \mathrm{z}_{\mathrm{i}}\right)$ is the covariance between parent trait and the relative fitness and $\mathrm{E}_{\omega \iota}\left(\Delta \mathrm{z}_{\mathrm{i}}\right)$ is the fitnessweighed average of $\Delta \mathrm{z}_{\mathrm{i}}$, the difference between trait of the parent, and the mean trait of the offspring of parent $\mathrm{i}$. The difference from equation 3.1 is that now all fitnesses are relative, $\omega_{\mathrm{i}}$, instead of absolute, $\mathrm{w}_{\mathrm{i}}$.

There are several useful ways of further transforming the Price Equation. Below, we are mainly building our conceptualization on a partition presented by Okasha (2006) that decomposes change in population mean into three components.

$$
\text { (3.4) } \Delta \mathrm{z}=\mathrm{h} \operatorname{Cov}\left(\omega_{\mathrm{i}}, \mathrm{z}_{\mathrm{i}}\right)+\mathrm{E}\left(\Delta \mathrm{z}_{\mathrm{i}}\right)+\operatorname{Cov}\left(\omega_{\mathrm{i}}, \mathrm{e}_{\mathrm{i}}\right)
$$

The first term on the right hand side consists of $h$, the slope of the parent-offspring regression (or midparent offspring regression in the case of sexual reproduction; Okasha 2006) or the heritability of the trait value and the covariation between fitness and trait value of parental individual i, i.e., the selection differential. This first term is thus the well known breeder's equation (Falconer and Mackay 1996; Lynch and Walsh 1998): the change in population mean due to variation in parent fitness and the similarity between parents and offspring. The second term represents the change that is independent of fitness differences such as systematic change due to environmental differences or non-Mendelian genetics (Okasha 2006). The third term consists of the covariance between fitness of an individual in the parent generation and the residuals of the parent-offspring regression. The last two terms are usually thought to be unimportant for adaptive evolutionary change, and dropped in most population genetic approaches. Equation 3.3 can be expanded to include groupings just as in equation 3.1.

In evolutionary biology the Price Equation has mainly been used in the context of genetically inherited traits with simple underlying genetics. For example, applications of the equation have proved very successful for unifying theories of social evolution (Frank 1998; Okasha 2006), and for interpreting Fisher's fundamental theorem of natural selection (Frank 1997; Okasha 2006). Other recent applications include epidemiology and community ecology (summarized in Gardner 2008; Kerr and Godfrey-Smith 2009). However, its generality makes it an ideal starting point for clarifying differences between systems of inheritance.

Our aim here is to use the Price Equation to conceptualize differences and similarities between the four classes of inheritance described by Jablonka and Lamb (2005). Based on this analysis, we propose a novel 
framework and use this as a discussion for the potential implications of different inheritance systems for the rate and direction of evolutionary change. We emphasize that our ambition here is only to outline the basic differences and similarities between inheritance systems using the Price Equation and introduce it as an interpretative framework for future work. We do not aim to theoretically model, or review models of, specific scenarios that may require more explicit mathematical approaches, and will always be sensitive to further assumptions that could differ within and between inheritance systems (see Lehmann 2007 and Lehmann et al. 2008 for recent examples).

\section{Systems of Inheritance and the Price Equation}

We proceed by building simple verbal examples to assess how different modes of inheritance may change direction and rate of phenotypic change in a population compared to a baseline of Modern Synthesis genetic inheritance, as captured in the breeder's equation; in terms of the Price Equation $\Delta \mathrm{z}=\mathrm{h}$ $\operatorname{Cov}\left(\omega_{i}, z_{i}\right)$. (These different types of inheritance are schematically represented in Figure 1A-H.) Under the reasonable assumption that inheritance mechanisms do not affect selection (i.e., the covariation of trait value and fitness in the current generation) on the time scale of a single generation, any differences must arise through effects on transmission (selection can of course result in changes in the covariance of fitness and trait value in future generations; Frank 1995). From equation 3.4 it is evident that if selection remains unchanged, there are two other factors that affect evolutionary change. First is the heritability of traits that affects the response to selection and second is biases in transmission of traits from one generation to the next. Throughout, we refer to parents and offspring in their biological meaning and evolution as changes in phenotypes from the parental generation to the offspring generation. An alternative partitioning of the Price Equation has been recently proposed by Kerr and Godfrey-Smith (Godfrey-Smith 2009; Kerr and GodfreySmith 2009). Their approach looks at coancestry from the perspective of the transmitted trait, and sees "parentage" as the link between individuals who acquire traits, and the sources of these traits, whether there are pedigree links or not. While it is clear that this approach is a useful way of addressing evolutionary change in ways other partitions do not allow, we maintain our perspective since it allows easy comparison to the breeder's equation and the classification of inheritance systems according to Jablonka and Lamb (2005).

\subsection{Genetic inheritance: the traditional view}

Because parents and offspring share genes underlying trait values, parents and offspring are more similar to each other than individuals in the population are on average. Under genetic inheritance the direction of non-random change is affected by selection (Figure 1A), i.e. the covariance of fitness and phenotype or Cov $\left(\omega_{i}, z_{i}\right)$ in equation 3.4. The magnitude of change due to selection depends on the heritability (h in equation 3.4) and a weak correlation between parent $i$ and offspring genotypes i' may hinder evolutionary change even if selection is strong. This is the left hand column in Table 1; the baseline to which other inheritance mechanisms will be compared. For handling complexities in the Price Equation arising from sexual reproduction, such as allelic dominance effects, see Rice (2004) and Heywood (2005).

Under vertical genetic transmission, additive genetic variance underlying the trait, and a population without group structuring, the second right hand term in equation $3.4\left[\mathrm{E}\left(\Delta \mathrm{z}_{\mathrm{i}}\right)\right]$ does not affect the direction of evolutionary change in a single generation in a systematic way, even if chance events such as drift and mutation occur. This is because mutations occur at random (Figure 1B) and their effect on the population trait mean will average out in a large population. Transmission is therefore unbiased with regards to trait value and its fitness effect. If we also make the reasonable assumption that the third right hand term in equation $3.4\left[\operatorname{Cov}\left(\omega_{\mathrm{i}}, \mathrm{e}\right)\right]$ is zero under genetic inheritance, similarity of trait values between parent and offspring does not depend on the trait value itself, or its covariation with fitness, and all directional change will be due to selection. However, predictable single generation changes in the trait value are possible if the genetic architecture of the trait is complex (Heywood 2005), or if not all phenotypic effects of mutations are equally likely, again depending on the genetic architecture of the trait. 
A

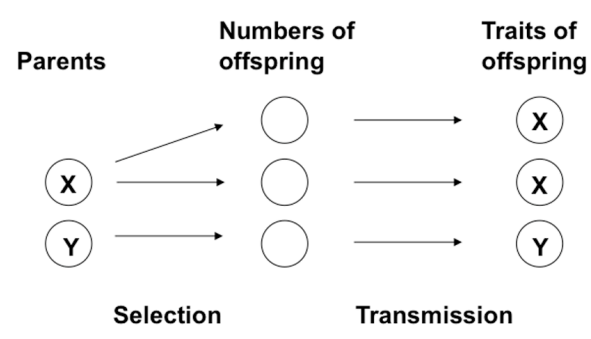

C

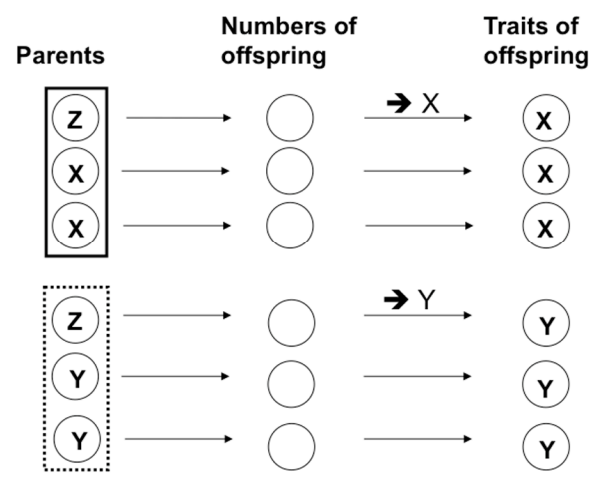

$E$

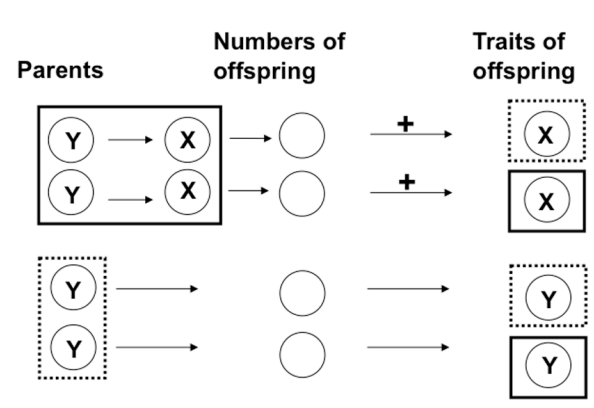

G

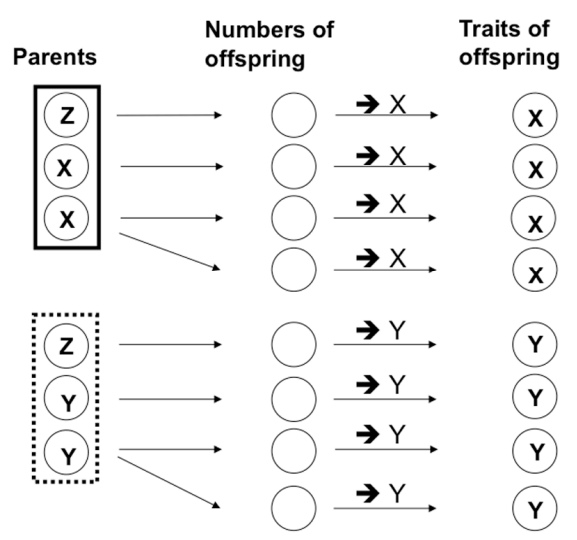

\&)
B

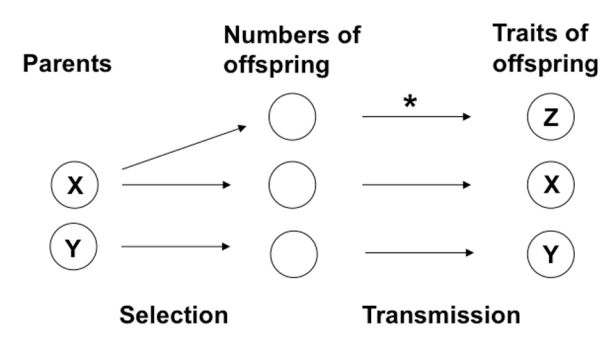

D

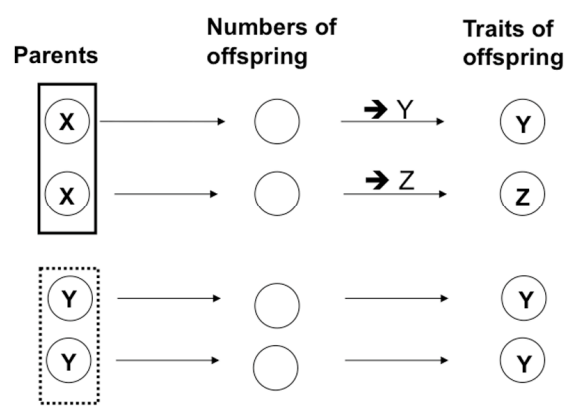

\section{$\mathrm{F}$}

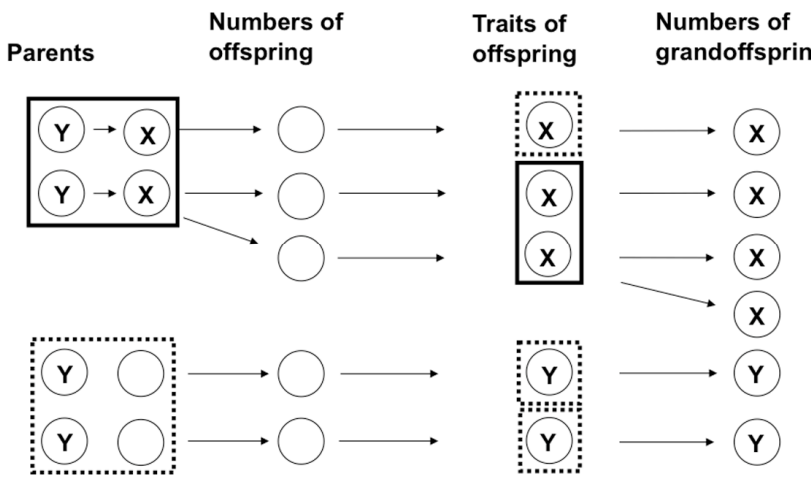

$\mathrm{H}$

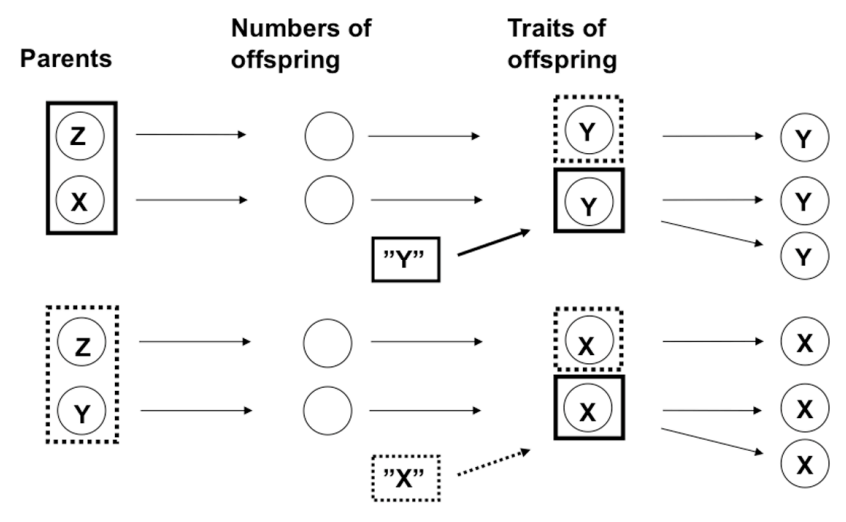


Figure 1 - Schematic Representations of the Different Types of Inheritance

Legend: Circles in the first column represent parental individuals, with trait values denoted by the letters; circles in the second column represent the numbers of offspring for each parent after selection; circles in the third column show the trait values of these offspring after transmission. When present, the fourth column denotes number of grand offspring after reproduction and selection on the offspring generation.

\section{A: Genetic vertical transmission with perfect transmission fidelity}

Trait $\mathrm{x}$ has higher fitness than $\mathrm{y}$ (more offspring), and since each of these also carries phenotype $\mathrm{x}$, $\mathrm{x}$ increases in frequency due to selection.

\section{B: Genetic vertical transmission with random mutation}

Trait $\mathrm{x}$ has higher fitness than $\mathrm{y}$ (more offspring), but trait $\mathrm{z}$ appears in population due to a random mutation in offspring of $\mathrm{x}$ (marked with*). The mutation lowers transmission fidelity, and hinders response to selection. The mutant phenotype increases in frequency through transmission.

\section{G: Genetic horizontal acquisition, no selection}

Individuals acquire genetic material from the most common phenotype in their group ( $\mathrm{x}$ in upper, $\mathrm{y}$ in lower) and common phenotypes increase in frequency due to a systematic transmission bias. Applies also to imitative and non-imitative acquisition in cases where acquisition is not fitness biased (panel G).

\section{D: Genetic inheritance with vertical transmission and induced mutation}

Individuals in the upper group mutate randomly, which changes the population phenotype in a random direction. In the lower group, the environment does not induce mutations, and transmission fidelity remains high.

\section{E: Environmental induction of traits}

Environment of the upper group induces trait $\mathrm{x}$ in the parent, which is transmitted to offspring irrespective of their future environment. Applies to epigenetic variants, behavior inducing substances, and parental effects with the difference that under the two latter the induced trait does not necessarily appear in the parent, but possibly only in the offspring, marked with the + sign. The induced phenotype increases in frequency through transmission.

\section{F: Environmental induction of a trait, a positive fitness effect}

The environment of the upper group induces trait $\mathrm{x}$ in the parent, that confers a fitness benefit to the parent and is transmitted to offspring, and confers a fitness benefit to offspring living in the same environment. As in panel E, this applies also to behavior inducing substances and parental effects. Induced trait increases in frequency through selection and transmission.

\section{G: Imitative or non-imitative horizontal acquisition}

Individuals acquire a phenotype by copying from the fittest phenotype in their group ( $\mathrm{x}$ in upper, $\mathrm{y}$ in lower); fitter phenotypes increase in frequency through transmission.

\section{H: Symbolic acquisition from an external source}

Individuals in the offspring generation acquire a phenotype from an environment specific external "instruction" with a fitness benefit in the same environment. Fitter phenotypes increase in frequency through transmission.

Group structures may have important implications for evolution under genetic inheritance for at least two reasons. First, the optimal phenotype (i.e., direction of selection) in group structured populations depends on group composition, as formulated in inclusive fitness theory (e.g., Frank 1998; equation 3.2). Second, the phenotype of an individual may depend not only on the genes inherited from parents of the individual, but also on genes of other individuals in the group through indirect genetic effects (Wolf et al. 1998). Third, the environment may influence the response to selection by causing changes in the proportion of variance explained by additive genetic and environmental effects (Hoffmann and Merilä 1999). Such effects affect the narrow-sense heritability of a trait ( $\mathrm{h}$ in equation 3.4) and can be conceptualized as groupspecific transmission biases.

\subsection{Genetic inheritance: horizontal transmission}

In terms of the Price Theorem, horizontal transmission of genetic material decreases similarity of parents and offspring, because genetic material can be acquired from other individuals in the population (Figure 1C). Implications of horizontal transfer on the direction and rate of evolutionary change depend on the nature of transfer. At its simplest, horizontal transmission of genetic material has no other effect than decreasing the similarity (h) of trait values between parent and offspring, compared to strict vertical transmission (Table 1). All else being equal, this makes the response to selection smaller and thus affects the rate but not the direction of phenotypic change, and may induce a random change in the population by making the expectation of offspring trait value deviate from the parental value (Table 1). If the deviation is random, horizontal transmission is conceptually similar to random mutation above, i.e. it induces change that is blind with respect to trait value and fitness effect. Thus, it does not systematically affect the direction 
VERTICAL

TRANSMISSION

\begin{tabular}{|c|c|c|c|c|c|c|c|}
\hline $\begin{array}{c}\text { Price } \\
\text { Equation } \\
(1.3)\end{array}$ & $\begin{array}{c}\text { GENETIC, } \\
\text { TRADITIONAL }\end{array}$ & $\begin{array}{l}\text { EPIGENETIC, } \\
\text { STABLE } \\
\text { VARIANTS IN } \\
\text { SUBSEQUENT } \\
\text { GENERATIONS }\end{array}$ & $\begin{array}{l}\text { GENETIC, } \\
\text { INDUCED } \\
\text { MUTATION }\end{array}$ & $\begin{array}{c}\text { EPIGENETIC/ } \\
\text { MATERNAL }\end{array}$ & $\begin{array}{c}\text { BEHAVIORAL } \\
\text { THROUGH } \\
\text { INTIMATE } \\
\text { PARENT- } \\
\text { OFFSPRLNG } \\
\text { RELATIONSHIP }\end{array}$ & $\begin{array}{c}\text { GENETIC, } \\
\text { HORIZONTAL }\end{array}$ & LEARNING \\
\hline $\operatorname{Cov}\left(w_{i}, z_{i}\right)$ & \multicolumn{7}{|c|}{ no effect } \\
\hline $\mathrm{h}$ & $\begin{array}{l}\text { determined by } \\
\text { additive genetic } \\
\text { variation }\end{array}$ & $\begin{array}{l}\text { determined by } \\
\text { stability of } \\
\text { epialleles } \\
\text { within and } \\
\text { across } \\
\text { environments }\end{array}$ & $\begin{array}{c}\text { random } \\
\text { mutation } \\
\text { decreases } \\
\text { heritability }\end{array}$ & $\begin{array}{l}\text { decreases if pare } \\
\text { trait induced }\end{array}$ & $\begin{array}{l}t \text { did not have the } \\
\text { n the offspring }\end{array}$ & \multicolumn{2}{|c|}{$\begin{array}{l}\text { acquisition of traits from non-parents decreases } \\
\text { heritability }\end{array}$} \\
\hline $\begin{array}{c}\mathrm{E}\left(\Delta \mathrm{z}_{\mathrm{i}}\right) \\
\text { random } \\
\text { direction }\end{array}$ & $\begin{array}{l}\text { mutation, } \\
\text { non-additive } \\
\text { genetics }\end{array}$ & $\begin{array}{l}\text { determined by } \\
\text { stability of } \\
\text { epialleles } \\
\text { within and } \\
\text { across } \\
\text { environments }\end{array}$ & $\begin{array}{l}\text { induced, non- } \\
\text { directional } \\
\text { mutation makes } \\
\text { this non zero }\end{array}$ & $\begin{array}{r}\text { if environmen } \\
\text { develop }\end{array}$ & tal effects make & \multicolumn{2}{|c|}{ if trait acquisition is random } \\
\hline $\begin{array}{c}\mathrm{E}\left(\Delta \mathrm{z}_{\mathrm{i}}\right) \\
\text { predictable } \\
\text { direction }\end{array}$ & $\begin{array}{l}\text { non-additive } \\
\text { genetics, biased } \\
\text { mutational } \\
\text { effects }\end{array}$ & $\begin{array}{l}\text { depends on the } \\
\text { stability of the } \\
\text { variants and } \\
\text { the environment }\end{array}$ & $\begin{array}{c}\text { biased } \\
\text { mutational } \\
\text { effects }\end{array}$ & $\begin{array}{l}\text { if each envir } \\
\text { predictal }\end{array}$ & $\begin{array}{l}\text { nment induces } \\
\text { le variants }\end{array}$ & \multicolumn{2}{|c|}{ if acquisition is based on frequency } \\
\hline $\begin{array}{c}\mathrm{E}\left(\Delta \mathrm{z}_{\mathrm{i}}\right) \text { fitness } \\
\text { increasing } \\
\text { direction }\end{array}$ & unlikely & $\begin{array}{l}\text { depends on the } \\
\text { stability of the } \\
\text { variants and } \\
\text { their fitness } \\
\text { effects in the } \\
\text { environment }\end{array}$ & unlikely & $\begin{array}{r}\text { if each envir } \\
\text { variants that in } \\
\text { that en }\end{array}$ & $\begin{array}{l}\text { nment induces } \\
\text { eease the fitness in } \\
\text { ironment }\end{array}$ & $\begin{array}{l}\text { if based on } \\
\text { frequency, and } \\
\text { frequency } \\
\text { reliably reflects } \\
\text { fitness }\end{array}$ & if acquisition rules based on fitness \\
\hline $\operatorname{Cov}\left(w_{i}, e_{i}\right)$ & \multicolumn{6}{|c|}{ unlikely } & $\begin{array}{l}\text { if the propensity to acquire traits } \\
\text { depends on parental fitness }\end{array}$ \\
\hline $\begin{array}{l}\text { The role of } \\
\text { grouping }\end{array}$ & $\begin{array}{r}\text { incorporation } \\
\text { selection and ina } \\
\text { of group memb }\end{array}$ & $\begin{array}{l}f \text { multi-level } \\
\text { ect genetic effects } \\
\text { s on individual } \\
\text { ype }\end{array}$ & & $\begin{array}{l}\text { groups define differe } \\
\text { nducing environmen }\end{array}$ & & \multicolumn{2}{|c|}{$\begin{array}{l}\text { groups define differences in the possible array of traits } \\
\text { that can be acquired }\end{array}$} \\
\hline
\end{tabular}

Table 1 - Classification of inheritance mechanisms (vertical transmission, induction, and acquisition) relative to the terms in the Price Equation

of evolutionary change through a transmission bias $\left[\mathrm{E}\left(\Delta \mathrm{z}_{\mathrm{i}}\right)\right]$, but by weakening similarity between offspring and parents it may reduce the response to selection.

However, horizontal transmission of genetic material can be more complex and transmission can be biased in ways that affect direction and rate of phenotypic change (Table 1; Figure 1C). For example, if not all genotypes are transmitted at the same rate, the probability of horizontal acquisition could be biased towards certain genotypes, which become more and more common. Thus, predictable directional change without selection is a relevant possibility, and whether this change increases the fitness of individuals depends on whether the commonly acquired traits are the ones with positive fitness effects. Finally, since individuals may acquire genes from other individuals in the population than their biological parents, offspring trait values must be greatly affected by the potential pool of non-parental "donor" individuals. If 
the possible trait values vary in a structured way within a population, this can be incorporated in the Price Equation as transmission biases that vary among groups (equation 3.2, Table 1), corresponding to the population structure.

\subsection{Induced mutation}

Similar to "normal" chance mutation and unbiased horizontal transmission, mutation induced by environmental conditions does not affect the direction of change in the population towards or away from more fit phenotypes, but since it weakens the parent-offspring correlation, it may slow down the response to selection (Table 1). This is true as long as the effects of mutations on the trait value are not biased with respect to fitness effects of the traits.

If not all individuals in the population experience the inducing conditions, the transmission effects [E $\left(\Delta \mathrm{z}_{\mathrm{i}}\right)$ can be group specific (Figure 1D), and incorporated into the expanded form of the theorem with different groups (Table 1). Induced mutations are unlikely to affect the covariance between fitness and error in phenotype inheritance $\operatorname{Cov}\left(\omega_{\mathrm{i}}, \mathrm{e}\right)$. Thus, also under induced mutation, directional, predictable change in population is likely to be due to selection only.

\subsection{Epigenetic inheritance}

Epigenetic inheritance has a dual effect on evolutionary change over a single generation. First, epigenetic inheritance decreases $h$, the similarity between parent (the trait value of the parent before environmental induction of the epigenetic variant) and offspring phenotype, and thus can reduce the phenotypic response to selection (Table 1). Second, epigenetic variants cause a change in the population due to transmission biases $\left[\mathrm{E}\left(\Delta \mathrm{z}_{\mathrm{i}}\right)\right.$ (Table 1). This is because the parental phenotype or grouping of phenotypes may have specific effects on the offspring phenotype. Phenotypic variants induced by the parental environment (Figures 1E, F) can be incorporated into an expanded form of the Price Equation as transmission biases based on grouping of parents of individuals according to their environment. This is similar to induced mutation above and therefore classified together in Table 1. However, in the previous case, a mutation-inducing environment causes offspring with induced mutation change in a random direction. In the case of epigenetic variants, environment-specific variants are likely JJablonka and Lamb 1995), in which case the Price Equation needs to be expanded to incorporate group specific transmission biases.

As above, under epigenetic inheritance it is difficult to see a biological scenario where epigenetic effects would lead to the term $\operatorname{Cov}\left(\omega_{\mathrm{i}}\right.$, e) being different from zero. Thus, non-random change across two generations will be determined by the combined effects of selection and biased transmission. If epigenetic variants that are transmitted are the ones with positive fitness effects, epigenetic inheritance may augment the change due to selection, and increase the rate of adaptive evolutionary change in the population. Furthermore, similar to horizontal transmission of traits, directional change in a single generation is possible in the absence of a response to selection in the traditional sense.

Inherited epigenetic variants are conceptually very similar to maternal effects (Figures 1E, F; Badyaev and Uller 2009; Mousseau and Fox 1998; Uller 2008). In both, the phenotype or environment (grouping) of the parent affects the phenotype of the offspring in a way that is not explained by genetic change, and this can either enhance offspring fitness (e.g., via adaptive maternal effects; Figure 1H) or impede it (e.g., by the effects of poor maternal condition on offspring traits; Marshall and Uller 2007; Uller 2008). As with maternal effects more generally, epigenetic variants may be inherited over multiple generations even if the inducing environment changes, i.e., a trait induced by environment of a grandparent can be present in grand offspring even if the intermediate parent did not experience the inducing conditions (Morgan et al. 1999). In such cases, the inherited epigenetic variants may behave like genetic variants under vertical transmission. 


\subsection{Behavioral Inheritance}

In Jablonka and Lamb's classification, behavioral inheritance can occur through three distinct routes, each conceptually quite different, so we will deal with them separately.

Inheritance through behavior inducing substances: This class of inheritance is qualitatively similar to epigenetic inheritance or maternal effects above (Figures 1E, F). Induction of variants may weaken the correlation between parent and offspring genotypes because a parent may transmit a trait she did not have originally, and thus reduce the magnitude of the change due to selection (Table 1). However, there may be directional changes due to biased transmission, which again can differ between groups in different environments (Table 1).

Imitative social learning: In this class of behavioral inheritance individuals acquire traits from any other individual in the population, not necessarily parents (Avital and Jablonka 2000; Jablonka and Lamb 2005; Shea 2009). This allows a great degree of horizontal transmission (Figures 1C, G). As with horizontal transfer of genetic material above, this decreases similarity of parents and offspring and, as a consequence, the response to selection. As with maternal effects, transmission of traits may be biased towards certain trait values, e.g., due to their fitness effects (Figure 1G), or other effects the learning individual may observe or assess (e.g., Lehmann and Feldman 2008; Richerson and Boyd 2005). These effects are unlikely to work through $\operatorname{Cov}\left(\omega_{\mathrm{i}}, \mathrm{e}\right)$, so the Price Equation needs to be expanded by incorporating different types of trait acquisition into the term $\mathrm{E}\left(\Delta \mathrm{z}_{\mathrm{i}}\right)$. Through such effects, the population may undergo evolutionary change, even if there is no selection (see, e.g., Lehmann et al. 2008). Similar to the horizontal transfer of genes, the evolutionary change due to transmission depends on the directionality of transfer, such as whether transmission is biased towards trait values with positive fitness effects, which would mean that change due to transmission coincides with the response due to selection.

Non-imitative social learning: Though mechanistically different, this class of inheritance is very similar to imitative social learning in a qualitative sense (Figures 1C, G; Table 1), and analogous to other forms of horizontal acquisition of phenotypes which do not necessarily involve learning (Ehrlich and Levin 2005; Henrich and Boyd 1998; Richerson and Boyd 2005). The important factors are the group structures that determine the range of traits an individual may potentially acquire and the possibility of horizontal transmission, potentially with transmission bias towards trait values with positive fitness effects.

\subsection{Symbolic Inheritance}

The final class of inheritance mechanisms listed by Jablonka and Lamb is symbolic inheritance. Horizontal transmission also may be very common under this class, and similarity of parents and offspring thus low. This reduces the change due to selection, and consequently most change is happening due to transmission (Table 1). Under symbolic inheritance, the trait values that can be acquired are not limited to traits of other individuals currently present in the population (Figure 1H). Apart from that, symbolic inheritance is captured in the same way in the Price Equation as horizontal transmission in behavioral inheritance systems (Section 4.5; Table 1).

\section{Discussion}

We have used one of the possible partitions of the Price Equation to highlight conceptual differences and similarities of a recently proposed categorization of inheritance systems (Jablonka and Lamb 2005), and their implications for short term evolutionary change. Our analysis suggests that the four inheritance systems (genetic, epigenetic, behavioral and symbolic) do not form logically coherent and distinct clusters with respect to their effect across a single generation. Instead, our discussion of how they will be treated in the Price Equation suggests that they fall into three categories, each with potentially unique consequences for 
phenotypic change across generations (Table 1). For discussion purposes, we have named them "vertical transmission" (i.e., when traits are transmitted from parent to offspring), "induction" (when the environment determines change between parent and offspring) and "acquisition" (when traits originate from non-parental individuals or other sources). This conceptual classification complements the more mechanistic classification proposed by Jablonka and Lamb (2005). Our classification allows comparing short term evolutionary implications of different inheritance modes, and helps us point out links to other frameworks.

Three main points are highlighted by our analysis. First, the direction and strength of selection $\left[\operatorname{Cov}\left(\omega_{i}\right.\right.$, $\mathrm{z}_{\mathrm{i}}$ ] remain unchanged under vertical transmission, induction and acquisition. This is in line with one prevalent strategy of evolutionary modeling, optimization under the "phenotypic gambit" (Grafen 1984), where only phenotypic states are analyzed under the assumption that the inheritance system does not impose a constraint on selection so that the population in the long run responds to selection and the population mean moves towards the individual optimum.

Second, it is clear that through their effects on the response to selection, the inheritance modes differ, sometimes substantially, with respect to the rate of directional evolutionary change. Compared to vertical genetic inheritance with purely additive genetic effects, induction and acquisition tend to weaken the response to selection because they undermine parent-offspring resemblance (Table 1). This does not affect direction of selection, just the rate of change, and can be incorporated in the quantitative genetics research framework (see, e.g., Bossdorf et al. 2008; Johannes et al. 2008).

Third, non-genetic inheritance can produce predictable, even adaptive, phenotypic change that is not due to response to selection in the traditional sense of the breeder's equation. This may occur simultaneously with selection, and therefore reinforce the magnitude of phenotypic change, but is possible even in the absence of fitness differences in the parents or in the absence of genetic variation in the population, through transmission biases towards certain phenotypes.

Different inheritance modes are likely to differ in the conditions under which they facilitate adaptive change. In general, adaptive change is possible if more fit individuals leave more offspring that vertically inherit the adaptive trait, and/or if induction or acquisition are biased towards traits with positive fitness effects (row "E( $\left.\Delta \mathrm{z}_{\mathrm{i}}\right)$ fitness increasing direction" in Table 1$)$. In the former case, there is direct selection on the focal trait. In the latter, there is, or is likely to have been, selection on the inheritance mechanism, and directional phenotypic change in a population is possible in the absence of selection on the focal trait. In cases like this, concentrating only on the focal trait $\mathrm{z}$ would give a false impression of the role of selection. Inheritance mechanisms have to be seen as dynamic, evolving features of the organism (Badyaev 2008, 2009) and the impact of different inheritance mechanisms on evolutionary change probably depends on whether the inheritance mechanisms allow adaptive acquisition or induction of traits. Below we discuss how different transmission mechanisms affect rate and direction of adaptive change (summarized in Table 1).

\subsection{Vertical Transmission}

The baseline for the discussion is the vertical, Mendelian genetic transmission of the Modern Synthesis. New variants, generated via mutation and recombination, are generally random with respect to their fitness effects, or at least unlikely to lead to adaptive change even if mutations are not completely random in their phenotypic effects. Furthermore, such random effects (row " $\mathrm{E}\left(\Delta \mathrm{z}_{\mathrm{i}}\right)$ random direction" in Table 1) are likely to average out in a large population, and not lead to large change in a population mean. Adaptive, directed acquisition of traits is not possible and phenotypic change proceeds through selection that culls unfit variants, with a rate determined by the strength of selection and fidelity of transmission. Phenotypic change may be complicated, depending on the genetic architecture of traits, including dominance and epistatic effects (Heywood 2005) and indirect genetic effects, i.e., effects of genes in the social environment on the phenotype of the focal individual (Wolf et al. 1998; Wolf et al. 2000). Inherited epigenetic variants, when not newly acquired (see above), may behave similarly, but their long term stability in natural environments is an open question (Bossdorf et al. 2008; Richards 2006; Uller 2008). 


\subsection{Induction}

A distinct type of inheritance emerging from our analysis occurs when the environment of the parent affects the phenotype of the offspring. This can be either via induced random changes such as induced mutation in genes underlying trait $\mathrm{z}$, transmitted changes in gene expression (epigenetic inheritance), or via environmentally induced transfer of a substance other than DNA from parents to offspring. In evolutionary ecology, the latter ones are referred to as parental or maternal effects (Mousseau and Fox 1998; Uller 2008).

These three types are all characterized by decreased heritability in the strict sense (equation 1.4), but they likely vary in what kinds of variants are induced, and in how stable the new variants are. Thus, their implications for adaptive evolution may also differ. First, facultatively induced mutation is unlikely to lead to fast change in population mean, because mutation occurs randomly with respect to direction of phenotypic effects and fitness, and the effects on the population mean are likely to balance out. However, induced mutation in offspring may be beneficial for the parent when the future environment is unpredictable and likely to differ from the parental environment (Badyaev 2005; Meyers and Bull 2002).

Under induced mutation, where the direction of change in offspring of a single parent is random, the change in mean phenotype may be less affected compared to when each parent induces a similar change in all offspring (West-Eberhard 2003). The latter is possible under other modes of induction, where a certain phenotype is induced by the parental environment. In these cases, biased transmission may also cause the population to evolve in a predictable direction, whereby the induction mechanisms can also be seen as adaptive, evolved complex traits (Badyaev 2008; Jablonka and Lamb 1995, 2005). Adaptive evolution of such inheritance systems is likely to be dictated by the level of environmental heterogeneity in relation to generation time that determines the concordance of selection on parents and offspring (Godfray 1995; Mock and Parker 1998; Parker et al. 2002; Uller 2008).

After the initial induction, inheritance of phenotypes may be stabilized provided the conditions do not change, and therefore essentially become part of a vertical transmission system (Badyaev and Uller 2009; Baldwin 1896; Waddington 1953, 1996; see Table 1). Unfortunately, our understanding of the stability of epigenetic inheritance across generations and environments in natural populations is very limited and research on the ecology of epigenetic effects is needed before we can evaluate its role in adaptive evolution (Bossdorf et al. 2008; Richards 2006).

\subsection{Acquisition}

The three types of inheritance we have classified as acquisition are different from vertical transmission and induction. First, phenotypic change in a population can be fast since any individual in the population is a potential source for trait acquisition or, in the case of symbolic inheritance, the source is not limited to individuals in current populations. The rate of change can therefore be high over a single generation either due to frequency dependent effects (see above for horizontal genetic transmission), or efficient copying of selectively favored variants. Especially in the cognition based acquisition of traits (cultural selection; CavalliSforza and Feldman 1981), this allows great flexibility and fast evolutionary change, even if selection [Cov $\left.\left(\omega_{i}, z_{i}\right)\right]$ is not necessarily directly involved in the change of the focal trait z. Another key difference to induction of traits is that in induction the external environment determines trait change, whereas under acquisition it is the distribution of traits in the evolving population (or symbolic instructions created by the population) itself that affects the traits of the next generation.

The key difference among the three acquisition mechanisms listed in Table 1 lies in the degree to which they enable directed and flexible acquisition or expression of traits. Acquisition of genetic material from other individuals in the population may be a facultative decision (Beaber et al. 2004), perhaps a result of adaptive evolution, but this does not allow for the acquisition of particular traits. Horizontal genetic transmission is thus similar to induced mutation producing change in an unpredictable direction, which may be adaptive under changing conditions (Badyaev 2005; Meyers and Bull 2002). However, for the diverse mechanisms of cultural acquisition of phenotypes, including learning and symbolic inheritance (Jablonka and Lamb 2005), it is obvious that the cognitively demanding acquisition rules are subject to selection, and 
may result in copying of adaptive, functional traits from a specific pool of variants available in the group or population. Adaptive learning is likely to lead to a link between the fitness of a trait and the degree to which it is acquired by members of the offspring generation (Henrich and Boyd 1998; Richerson and Boyd 2005; Weber and Depew 2003), which could result in rapid phenotypic change.

\section{Summary}

Our analysis suggests that the classification of inheritance systems into genetic, epigenetic, behavioral and symbolic, introduced by Jablonka and Lamb (2005), does not fully capture the evolutionarily differences between different means of inheritance. Our analysis identified similarities and differences between systems of inheritance that were not captured by previous classifications (summarized in Table 1), and introduces the Price Equation as a tool for the development and interpretation of more specific models of non-genetic inheritance. The mechanisms of inheritance do not affect the direction of selection, but they may nevertheless change how the population evolves in response to, or in the absence of, selection. Perhaps most importantly, the Price Equation clarifies that each mechanism of inheritance can be modified in several ways that are conceptually similar (Table 1), but differ in the extent to which they can produce rapid and directed phenotypic change. This should help to clarify under what circumstances different systems of inheritance actually impact on the classic Modern Synthesis formulation of the causes of evolutionary change. However, complementary approaches will be required to outline the long-term evolutionary consequences of different systems of inheritance (e.g., with respect to evolvability), and to assess the importance of complex interactions and feedbacks loops between within-generation change and selection that may occur when several mechanisms of inheritance are at work simultaneously (e.g., Feldman and Laland 1996; Lehmann and Feldman 2008; Odling-Smee et al. 2003; Richerson and Boyd 2005). Furthermore, although our approach does not directly address the origin, maintenance, and modification of inheritance systems, we argue that they are best seen as evolutionary stages in the construction and transfer of developmental resources across generations, the adaptive value of which will be set by the extent to which they enable the retention and reconstruction of reliable organismal-environment configurations (Badyaev and Uller 2009; Newman and Müller 2000; Newman and Müller 2006; Oyama 2000; Oyama et al. 2001).

\section{Literature cited}

Allis, C. D., T. Jenuwein and D. Reinberg. 2007. Epigenetics. Cold Spring Harbor Laboratory Press.

Avital, E. and E. Jablonka. 2000. Animal Traditions: Behavioural Inheritance in Evolution. Cambridge University Press.

Badyaev, A. 2008. Maternal effects as generators of evolutionary change. Annals of the New York Academy of Sciences 1133: 151-161.

Badyaev, A. and T. Uller. 2009. Parental effects in ecology and evolution: Mechanisms, processes, and implications. Philosophical Transactions of the Royal Society B: Biological Sciences 364: 1169-1177.

Badyaev, A. V. 2005. Stress-induced variation in evolution: from behavioural plasticity to genetic assimilation. Proceedings of the Royal Society B: Biological Sciences 272: 877-886.

Baldwin, J. M. 1896. A new factor in evolution. American Naturalist 30: 441-451.

Beaber, J. W., B. Hochhut and M. K. Waldor. 2004. SOS response promotes horizontal dissemination of antibiotic resistance genes. Nature 427: 72-74.

Bilkó, Á., V. Altbäcker and R. Hudson. 1994. Transmission of food preference in the rabbit: The means of information transfer. Physiology \& Behavior 56: 907-912.

Bjedov, I., O. Tenaillon, B. Gerard, V. Souza, E. Denamur, M. Radman, F. Taddei and I. Matic. 2003. Stress-induced mutagenesis in bacteria. Science 300: 1404-1409.

Bossdorf, O., G. L. Richards and M. Pigliucci. 2008. Epigenetics for ecologists. Ecology Letters 11: 106-115.

Boyd, R. and P. Richerson. 1985. Culture and the Evolutionary Process. University of Chicago Press.

Boyd, R. and P. Richerson. 2005. The Origin and Evolution of Cultures. Oxford University Press.

Caporale, L. 2000. Mutation is modulated: implications for evolution. BioEssays 22: 388-395. 
Cavalli-Sforza, L. and M. W. Feldman. 1981. Cultural Transmission and Evolution: A Quantitative Approach. Princeton University Press.

Crews, D., A. C. Gore, T. S. Hsu, N. L. Dangleben, M. Spinetta, T. Schallert, M. D. Anway and M. K. Skinner. 2007. Transgenerational epigenetic imprints on mate preference. Proceedings of the National Academy of Sciences of the United States of America 104: 5942-5946.

Crick, F. 1970. Central dogma of molecular biology. Nature 227: 561-563.

Cubas, P., G. Vincent and E. Coen. 1999. An epigenetic mutation responsible for natural variation in floral symmetry. Nature 401: 157-161.

Ehrlich, P. R. and S. A. Levin. 2005. The evolution of norms. PLoS Biology 3(6): e194.

Falconer, D. S. and T. F. C. Mackay. 1996. Introduction to Quantitative Genetics. Longman.

Feldman, M. W. and K. N. Laland. 1996. Gene-culture coevolutionary theory. Trends in Ecology \& Evolution 11: 453-457.

Frank, S. A. 1995. George Price's contributions to evolutionary genetics. Journal of Theoretical Biology 175: 373-388.

Frank, S. A. 1997. The Price Equation, Fisher's fundamental theorem, kin selection, and causal analysis. Evolution 51: 1712-1729.

Frank, S. A. 1998. Foundations of Social Evolution. Princeton University Press.

Franz, M. and C. L. Nunn. 2009. Rapid evolution of social learning. Journal of Evolutionary Biology 22: 1914-1922.

Futuyma, D. 2005. Evolutionary Biology. Sinauer Associates.

Gardner, A. 2008. The Price Equation. Current Biology 18: R198-R202.

Godfray, H. C. J. 1995. Evolutionary theory of parent-offspring conflict. Nature 376: 133-138.

Godfrey-Smith, P. 2009. Darwinian Populations and Natural Selection. Oxford University Press.

Gorelick, R. and M. D. Laubichler. 2008. Genetic $=$ Heritable (Genetic $\neq$ DNA). Biological Theory 3: 79-84.

Grafen, A. 1984. Natural selection, kin selection and group selection. In Behavioural Ecology. An Evolutionary Approach. Ed. by J. R. Krebs and N. B. Davies. Blackwell Scientific.

Haig, D. 2007. Weismann rules! OK? Epigenetics and the Lamarckian temptation. Biology \& Philosophy 22: 415-428.

Henrich, J. and R. Boyd. 1998. The evolution of conformist transmission and the emergence of betweengroup differences. Evolution and Human Behavior 19: 215-241.

Heywood, J. S. 2005. An exact form of the breeder's equation for the evolution of a quantitative trait under natural selection. Evolution 59: 2287-2298.

Hoffmann, A. A. and J. Merilä. 1999. Heritable variation and evolution under favourable and unfavourable conditions. Trends in Ecology and Evolution 14: 96-101.

Jablonka, E. and M. J. Lamb. 1995. Epigenetic Inheritance and Evolution: The Lamarckian Dimension. Oxford University Press.

Jablonka, E. and M. J. Lamb. 2005. Evolution in Four Dimensions: Genetic, Epigenetic, Behavioral, and Symbolic Variation in the History of Life. MIT Press.

Jablonka, E. and G. Raz. 2009. Transgenerational epigenetic inheritance: prevalence, mechanisms, and implications for the study of heredity and evolution. Quarterly Review of Biology 84: 131-176.

Johannes, F., V. Colot and R. C. Jansen. 2008. Epigenome dynamics: a quantitative genetics perspective. Nature Reviews Genetics 9: 883-890.

Kerr, B. and P. Godfrey-Smith. 2009. Generalization of the Price Equation for evolutionary change. Evolution 63: 531-536.

Laland, K. N. 2004. Social learning strategies. Learning \& Behavior 32: 4-14.

Leadbeater, E. and L. Chittka. 2008. Social transmission of nectar-robbing behaviour in bumble-bees. Proceedings of the Royal Society B: Biological Sciences 275: 1669-1674.

Lehmann, L. 2007. The evolution of trans-generational altruism: kin selection meets niche construction. Journal of Evolutionary Biology 20: 181-189.

Lehmann, L. and M. W. Feldman. 2008. The co-evolution of culturally inherited altruistic helping and cultural transmission under random group formation. Theoretical Population Biology 73: 506-516. 
Lehmann, L., M. W. Feldman and K. R. Foster. 2008. Cultural transmission can inhibit the evolution of altruistic helping. American Naturalist 172: 12-24.

Lynch, M. and B. Walsh. 1998. Genetics and Analysis of Quantitative Traits. Sinauer.

Mameli, M. 2004. Nongenetic selection and nongenetic inheritance. British Journal for the Philosophy of Science 55: 35-71.

Mameli, M. 2005. The inheritance of features. Biology \& Philosophy 20: 365-399.

Marshall, D. J. and T. Uller. 2007. When is a maternal effect adaptive? Oikos 116: 1957-1963.

Martienssen, R. A. and V. Colot. 2001. DNA methylation and epigenetic inheritance in plants and filamentous fungi. Science 293: 1070-1074.

Maynard Smith, J. and E. Szathmáry. 1995. The Major Transitions in Evolution. Oxford University Press.

Mayr, E. and W. B. Provine. (eds) 1980. The Evolutionary Synthesis. Perspectives in the Unification of Biology. Harvard University Press.

Mayr, E. 1982. The Growth of Biological Thought. Diversity, Evolution and Inheritance. Belknap Press, Harvard.

Meyers, L. A. and J. J. Bull. 2002. Fighting change with change: adaptive variation in an uncertain world. Trends in Ecology \& Evolution 17: 551-557.

Mock, D. W. and G. A. Parker. 1998. Siblicide, family conflict and the evolutionary limits of selfishness. Animal Behaviour 56: 1-10.

Molinier, J., G. Ries, C. Zipfel and B. Hohn. 2006. Transgeneration memory of stress in plants. Nature 442: 1046-1049.

Morgan, H. D., H. G. E. Sutherland, D. I. K. Martin and E. Whitelaw. 1999. Epigenetic inheritance at the agouti locus in the mouse. Nature Genetics 23: 314-318.

Mousseau, T. A. and C. W. Fox. 1998. Maternal Effects as Adaptations. Oxford University Press.

Newman, S. A. and G. Müller. 2000. Epigenetic mechanisms of character origination. Journal of Experimental Biology 288: 304-317.

Newman, S. A. and G. B. Müller. 2006. Genes and form: inherency in the evolution of developmental mechanisms. In Genes in Development: Rereading the Molecular Paradigm Ed. by E. M. NeumannHeld and C. Rehmann-Sutter. Duke University Press.

Ochman, H., J. G. Lawrence and E. A. Groisman. 2000. Lateral gene transfer and the nature of bacterial innovation. Nature 405: 299-304.

Odling-Smee, F. J., K. N. Laland and M. W. Feldman. 2003. Niche Construction: The Neglected Process in Evolution. Princeton University Press.

Okasha, S. 2006. Evolution and the Levels of Selection. Oxford University Press.

Oyama, S. 2000. The Ontogeny of Information: Developmental Systems and Evolution. Duke University Press.

Oyama, S., P. E. Griffiths and R. D. Gray. (eds) 2001. Cycles of Contingency: Developmental Systems and Evolution. MIT Press.

Parker, G. A., N. J. Royle and I. R. Hartley. 2002. Intrafamilial conflict and parental investment: a synthesis. Philosophical Transactions of the Royal Society B: Biological Sciences 357: 295-307.

Pigliucci, M. 2001. Phenotypic Plasticity: Beyond Nature and Nurture. Johns Hopkins University Press.

Pigliucci, M. 2007. Do we need an extended evolutionary synthesis? Evolution 61: 2743-2749.

Price, G. R. 1970. Selection and covariance. Nature 227: 520-521.

Price, G. R. 1972. Extension of covariance selection mathematics. Annals of Human Genetics 35: 485-490.

Provine, W. B. 2001. The Origins of Theoretical Population Genetics. University of Chicago Press.

Rando, O. J. and K. J. Verstrepen. 2007. Timescales of genetic and epigenetic inheritance. Cell 128: 655-668.

Rapp, R. A. and J. F. Wendel. 2005. Epigenetics and plant evolution. New Phytologist 168: 81-91.

Rice, S. H. 2004. Evolutionary Theory: Mathematical and Conceptual Foundations. Sinauer Associates.

Richards, E. J. 2006. Inherited epigenetic variation - revisiting soft inheritance. Nature Reviews Genetics 7: 395-401.

Richerson, P. and R. Boyd. 2005. Not by Genes Alone: How Culture Transformed Human Evolution. University of Chicago Press. 
Sarkar, S. 2006. From genes as determinants to DNA as resource: historical notes on development and DNA. In Genes in Development: Rereading the Molecular Paradigm. Ed. by E. M. Neumann-Held and C. Rehmann-Sutter. Duke University Press.

Shea, N. 2009. Imitation as an inheritance system. Philosophical Transactions of the Royal Society B: Biological Sciences 364: 2429-2443.

Tenaillon, O., E. Denamur and I. Matic. 2004. Evolutionary significance of stress-induced mutagenesis in bacteria. Trends in Microbiology 12: 264-270.

Uller, T. 2008. Developmental plasticity and the evolution of parental effects. Trends in Ecology \& Evolution 23: 432-438.

Varmuza, S. 2003. Epigenetics and the renaissance of heresy. Genome 46: 963-967.

Waddington, C. H. 1953. Genetic assimilation of an acquired character. Evolution 7: 118-126.

Waddington, C. H. 1996. Canalization of development and the inheritance of acquired characters. In Adaptive Individuals in Evolving Populations: Models and Algorithms. Ed. by R. K. Belew and M. Mitchell. Addison-Wesley Longman Publishing Co., Inc.

Weber, B. H. and D. J. Depew. (eds) 2003. Evolution and Learning. The Baldwin Effect Reconsidered. MIT Press.

Wolf, J. B., E. D. Brodie III, J. M. Cheverud, A. J. Moore and M. J. Wade. 1998. Evolutionary consequences of indirect genetic effects. Trends in Ecology \& Evolution 13: 64-69.

Wolf, J. B., E. D. Brodie III and M. J. Wade. 2000. Epistasis and the evolutionary process. Oxford University Press

\section{AGKNOWLEDGMENTS}

The authors would like to thank Andy Gardner, Kevin Foster and anonymous referees for comments, and all the authors who sent us their unpublished manuscripts for their help. HH was funded by the Academy of Finland (grants 121078, 213821).

Copyright (C) 2010 Author(s).

This is an open-access article distributed under the terms of the Creative Commons Attribution-NonCommercial-NoDerivs license, which permits anyone to download, copy, distribute, or display the full text without asking for permission, provided that the creator(s) are given full credit, no derivative works are created, and the work is not used for commercial purposes.

ISSN 1949-0739 